wickelt. Beseitigung des Spannungspneumothorax durch Bülaudrainage, die jedoch die Lungenatelektase nicht behob wegen Luftverlusten durch Bronchusabriß. Begleitende Brüche des Schlüsselbeins, Schulterblatts, der 1.-5. Rippe und Phrenicusparese rechts. Primäre Bronchusanastomose am 4. Tag nach Unfall führte zur vollen Ausdehnung der Lunge. Fortbestehen der Phrenicusparese 9 Monate nach Unfall.

Schliisselwörter: Bronchusabriß - Phrenicusparese - Claviculafraktur - Primäre Bronchusanastomose.

\title{
235. Diagnostik und Behandlung akuter thorakaler Aortenerkrankungen und -verletzungen
}

\author{
W. J. Stelter, M. Krüger und H. M. Becker \\ Chirurgische Klinik und Poliklinik der Universität München, Klinikum Großhadern \\ (Dir.: Prof. Dr. G. Heberer), Marchioninistraße 15, D-8000 München 70
}

\section{Diagnosis and Treatment of Acute Diseases and Ruptures of the Thoracic Aorta}

Summary. From 1973 to 1983,87 patients with diseases of the thoracic aorta, exclusively the aorta ascendens, (15 acute and chronic dissection, 53 degenerative or chronic-traumatic aneurysms, 19 acute traumatic ruptures) have been operated upon. Of the 87 patients, 54 required urgent or emergency operations because of impending or completed rupture; 21 patients $(39 \%$ ) died postoperatively. For diagnosis in suspected aortic disease, angiocomputer tomography has proved to be very useful as a first measure, followed by angiography for delineation of the origin of the aortic branches.

Key words: Thoracic aorta - Computer tomography.

Zusammenfassung. Von 1973 bis 1983 wurden 87 Patienten mit Erkrankungen der thorakalen Aorta, ausschließlich der Aorta ascendens (15 akute und chronische Dissektionen, 53 degenerative und chronischtraumatische Aneurysmen, 19 frische Verletzungen), operativ behandelt. 54 der 87 Eingriffe wurden als Notfalloder dringliche Operationen durchgeführt wegen drohender oder eingetretener Ruptur. 21 Patienten verstarben postoperativ, das entspricht $39 \%$. Für die Diagnostik hat sich als Erstmaßnahme bei Verdacht auf eine Aortenerkrankung die Angio-Computertomographie bewährt. Erst sekundär wird angiographiert, die Verhältnisse an den Abgängen der Aaorta dargestellt.

Schliisselwörter: Thorakale Aorta - Computertomographie.

\section{Notfalldiagnostik und operatives Konzept bei der frischen traumatischen Zwerchfellruptur}

\author{
U. Albers, J. Voigt, H. Hamelmann und E. Bauer \\ Abteilung Allgemeine Chirurgie, Chirurgische Universitätsklinik Kiel, Hospitalstraße 40, D-2300 Kiel
}

\section{Early Diagnosis and Operative Concept of Fresh Diaphragmatic Hernia}

Summary. Thirty-three patients with traumatic diaphragmatic hernia due to blunt abdominal injury were treated from 1968 to 1982. The left side was involved in 28 cases and the right in 8 cases. Early diagnosis relies on careful history, physical examination and repeated chest roentgenograms. Insertion of a nasogastric tube and contrast studies confirmed a ruptured diaphragm before the operation. Repair of diaphragmatic rupture on the left side should be attempted as soon as possible by laparotomy incision and on the right side by thoracotomy. A double layer with absorbable suture material sufficed in all cases.

Key words: Diaphragmatic rupture - Diagnosis - Operative concept.

Zusammenfassung. In 15 Jahren (1968-1982) wurden 33 Patienten mit einer indirekten traumatischen Zwerchfellruptur (links 28, rechts 8 ) behandelt. Die Frühdiagnose beruht auf der sorgfältigen Anamnese, physikalischen Untersuchung und wiederholten Thoraxröntgenaufnahmen. Magensonde und Kontrastmitteluntersuchungen bestätigten den Verdacht auf eine Zwerchfellruptur präoperativ. Linksseitig sollte so bald wie möglich durch eine Laparotomie, rechtsseitig durch eine Thoracotomie operiert werden. Bewährt hat sich der zweireihige Verschluß des Zwerchfells mit resorbierbarem Nahtmaterial.

Schlüsselwörter: Zwerchfellruptur - Notfalldiagnose - Operatives Konzept. 